\title{
Effect of depth gradient and temperature on Zostera marina vegetative and reproductive phenology in the first year of the 1997-1998 EI Niño
}

\section{Efecto de la profundidad y la temperatura en la fenología reproductiva y vegetativa de Zostera marina durante el primer año de El Niño 1997-1998}

\author{
Alf Enrique Meling-López ${ }^{1}$, Silvia Emilia Ibarra-Obando ${ }^{2}$, Horacio de la Cueva ${ }^{3 *}$, \\ Pedro Ortega-Romero ${ }^{1}$, Adriana Leticia Navarro-Verdugo ${ }^{4}$ \\ ${ }^{1}$ Departamento de Investigaciones Científicas y Tecnológicas, Universidad de Sonora, Colosio s/n entre \\ Sahuaripa y Reforma, Col. Centro, CP 83000, Sonora, Mexico. \\ 2 Departamento de Ecología Marina, Centro de Investigación Científica y de Educación Superior de Ensenada, \\ Carretera Ensenada-Tijuana, no. 3918, Zona Playitas, CP 22860, Ensenada, Baja California, Mexico. \\ ${ }^{3}$ Departamento de Biología de la Conservación, Centro de Investigación Científica y de Educación Superior \\ de Ensenada, Carretera Ensenada-Tijuana, no. 3918, Zona Playitas, CP 22860, Ensenada, Baja California, \\ Mexico. \\ ${ }^{4}$ Departamento de Física, Matemáticas e Ingeniería, Universidad de Sonora, Unidad Regional Sur, Lázaro \\ Cárdenas, no. 100, Col. Francisco Villa, CP 85890, Navojoa, Sonora, Mexico.
}

* Corresponding author. E-mail: cuevas@cicese.mx

\begin{abstract}
Does Zostera marina exhibit phenotypic plasticity, maximizing fitness in traits responding to environmental factors, i.e., depth and temperature? We compared the vegetative and sexual phenology and reproductive effort of $Z$. marina by analyzing vegetative and reproductive shoot density, biomass, and reproductive stages to determine structural features of vegetative and reproductive shoots from subtidal and intertidal environments in San Quintín Bay, Baja California, a year before and after the 1997-1998 El Niño/Southern Oscillation (ENSO). We found significant differences in vegetative and reproductive biomass between intertidal and subtidal environments driven by temperature differences between ENSO and non-ENSO years. Subtidal plants had lower density of long reproductive shoots and a shorter reproductive cycle. Seed release occurred from May to October in the subtidal environment, and from May to November in the intertidal environment. Maximal recorded values were $219.5( \pm 45.8)$ seeds per reproductive shoot in the subtidal environment and $151.3( \pm 21.5)$ in the intertidal environment. We observed higher sexual activity and lower vegetative biomass in the intertidal environment, the most stressful environment. Both vegetative and reproductive biomass were affected by the increase in temperature during ENSO, but vegetative and reproductive shoot densities were not affected.
\end{abstract}

Key words: vegetative biomass, reproductive shoots, reproductive cycles, temperature gradients, shoot density.

RESUMEN. ¿Exhibe Zostera marina plasticidad fenotípica para maximizar la adecuación en características estructurales que responden a factores ambientales como la profundidad y la temperatura? Comparamos la fenología sexual y vegetativa y el esfuerzo reproductivo de Z. marina analizando la densidad, la biomasa y los estadios reproductivos de haces vegetativos y reproductivos para determinar las características estructurales de los haces vegetativos y reproductivos de ambientes submareales e intermareales en la bahía San Quintín, Baja California, un año antes y después de El Niño/Oscilación del Sur (ENOS) de 1997-1998. Encontramos diferencias significativas en la biomasa vegetativa y reproductiva entre el ambiente submareal y el ambiente intermareal que fueron forzadas por las diferencias de temperatura entre los años con ENOS y sin ENOS. Las plantas del submareal tuvieron menor densidad de haces reproductivos largos y un ciclo reproductivo más corto. La liberación de semillas se presentó de mayo a octubre en el submareal y de mayo a noviembre en el intermareal. Los valores máximos registrados fueron $219.5( \pm 45.8)$ semillas por haz reproductivo en el submareal y $151.3( \pm 21.5)$ en el intermareal. Observamos mayor actividad sexual y menor biomasa vegetativa en el intermareal, el ambiente más estresante. Tanto la biomasa vegetativa como la reproductiva fueron afectadas por un aumento en la temperatura durante el evento ENOS, pero las densidades de haces vegetativos y reproductivos no fueron afectadas.

Palabras clave: biomasa vegetativa, haces reproductivos, ciclos reproductivos, gradientes de temperatura, densidad de haces.

\section{INTRODUCTION}

Characterized by high growth dynamics and plasticity, Zostera marina is the most widely distributed and abundant marine plant in the Northern Hemisphere (Lee et al. 2006). It is found along the Pacific coast of North America, from the temperate Alaska coastal waters to the hot waters

\section{INTRODUCCIÓN}

Zostera marina es la planta marina más abundante y más ampliamente distribuida del hemisferio norte (Lee et al. 2006) y se caracteriza por su gran dinámica de crecimiento y su plasticidad. En las costas del Pacífico de Norteamérica se localiza desde las aguas templadas de Alaska hasta las 
in the Gulf of California (Riosmena-Rodríguez et al. 2013). Zostera marina grows in shallow bays, coastal lagoons, and estuaries, in subtidal and intertidal environments (RiosmenaRodríguez et al. 2013). Its upper distribution limit is determined by shoot tolerance to desiccation from air exposure, and its lower distribution limit by light penetration (CabelloPasini et al. 2002). At low tide, intertidal Z. marina populations are exposed to extreme light intensity, air, water temperature variations, and other factors like grazing by waterfowl, all stressful conditions for shoot growth, vegetative biomass growth, and sexual reproduction. The subtidal environment is more stable (Lee et al. 2006); here stress is caused by light penetration as a function of depth, illumination, and water turbidity (Nielsen et al. 2002).

Sexual reproduction may play a crucial role in the maintenance of $Z$. marina meadows. Plants allocate more energy to sexual reproduction in stressful or disturbed environments (Cabaço and Santos 2012, Kaul 2016), and increased water temperatures provide the right conditions for vegetative biomass growth and other demographic characteristics (Cabello-Pasini et al. 2002). Increased water temperature probably promotes the development of enhanced sexual phenological characteristics; however, long periods of high temperature or exceptional events of extreme temperature decompensate the respiration/photosynthesis rate and carbohydrates are not passed on to physiological functions. This has strong negative effects on the number of leaves per shoot and reduces shoot biomass (Hammer et al. 2018).

San Quintín Bay (SQB) is relatively pristine, but some ecological processes there have an uncertain future, for example water turbidity, which increased by 2 -fold over the last 2 decades (Cabello-Pasini et al. 2003). SQB represents the southern limit of the central portion of the $Z$. marina distribution range (Phillips et al. 1983). Here Z. marina beds grow in areas that leave them exposed during low tides. Ibarra-Obando et al. (1997) and Poumian-Tapia and Ibarra-Obando (1999) have described several structural and functional characteristics of intertidal $Z$. marina beds in SQB. Differences in environmental conditions lead to differences in biomass, morphological characteristics, and depth distribution. Echavarria-Heras et al. (2006) compared Z. marina leaf morphology and biomass before and after the 1986-1987 El Niño/Southern Oscillation (ENSO) event and found differences for most of the measured variables; these differences could be explained by the warm water associated with the ENSO event.

The vegetative and sexual biomass, shoot density, shoot length, and reproductive effort of $Z$. marina growing within a depth gradient ranging from the shallow subtidal to the intertidal areas of SQB have never been compared simultaneously, nor have they been assessed during an ENSO event. Information on Z. marina vegetative biomass and sexual phenology associated with reproductive effort is scarce and fragmented, and so is information on how this species is affected by unusual high-temperature events such as ENSO aguas cálidas del golfo de California (Riosmena-Rodríguez et al. 2013). La especie crece en bahías someras, lagunas costeras y estuarios, tanto en ambientes intermareales como submareales (Riosmena-Rodríguez et al. 2013). El límite de su distribución sobre el intermareal está determinado por la tolerancia de sus haces a la desecación debido a la exposición al aire, mientras que su profundidad máxima está limitada por la penetración de la luz (Cabello-Pasini et al. 2002). Durante las mareas bajas, las poblaciones de Z. marina del intermareal están expuestas a intensidad de luz extrema, aire y variaciones de la temperatura del agua, así como a otros factores como el pastoreo por aves acuáticas cinegéticas, todas condiciones estresantes para el crecimiento de los haces, el incremento de biomasa vegetativa y la reproducción sexual. El submareal es un ambiente más estable (Lee et al. 2006), donde el estrés está determinado por la penetración de la luz como función de la profundidad, la iluminación y la turbidez del agua (Nielsen et al. 2002).

La habilidad de Z. marina para reproducirse sexualmente podría ser crucial para el mantenimiento de sus praderas. Las plantas asignan más energía a la reproducción sexual en ambientes estresantes o perturbados (Cabaço y Santos 2012, Kaul 2016), y los incrementos en la temperatura del agua crean condiciones propicias para el incremento de biomasa vegetativa y otras características demográficas (CabelloPasini et al. 2002). El aumento de la temperatura del agua probablemente promueve el desarrollo de mejores características en la fenología sexual; sin embargo, los periodos largos de exposición a altas temperaturas o eventos excepcionales de temperaturas extremas descompensan la tasa de respiración/fotosíntesis y los carbohidratos ya no intervienen en los procesos fisiológicos. Esto tiene un fuerte efecto negativo en el número de hojas por haz y reduce la biomasa de los haces (Hammer et al. 2018).

La bahía San Quintín (BSQ) es relativamente prístina, pero algunos de los procesos ecológicos ahí tienen un futuro incierto, por ejemplo la turbidez del agua, que se duplicó en las últimas 2 décadas (Cabello-Pasini et al. 2003). La BSQ representa el límite sur de la porción central del intervalo de distribución de Z. marina (Phillips et al. 1983). En $\mathrm{BSQ}$, las praderas de $Z$. marina también se desarrollan en sitios expuestos durante las mareas bajas. Ibarra-Obando et al. (1997) y Poumian-Tapia e Ibarra-Obando (1999) han descrito diversas características estructurales y funcionales de las praderas de Z. marina del intermareal de BSQ. Las diferencias en las condiciones ambientales promueven diferencias en la biomasa, las características morfológicas y la distribución en la profundidad. Echavarria-Heras et al. (2006) compararon la biomasa y la morfología de las hojas de Z. marina antes y después del evento de El Niño/ Oscilación del Sur (ENOS) de 1986-1987 y encontraron diferencias en muchas de las variables que midieron; las diferencias podrían ser explicadas por la temperatura más cálida del agua asociada al evento ENOS. 
or within a depth gradient. We hypothesize that the water temperature increase that took place from 1996 to the $1997-$ 1998 ENSO period resulted in reduced vegetative biomass and increased sexual phenology for Z. marina. The best environment for reproductive phenology is the intertidal zone, whereas the subtidal zone is best for developing vegetative characteristics.

With the advent of climate change, we need deeper knowledge on the behavior of $Z$. marina meadows. If extreme temperature events become more frequent and sea level changes the norm, we must be able to predict how the species will respond.

\section{MATERIALS AND METHODS}

SQB is a coastal lagoon $\left(30^{\circ} 30^{\prime} \mathrm{N}, 116^{\circ} 01^{\prime} \mathrm{W}\right)$ on the Pacific coast of Baja California, Mexico. Its community ecology has been described by Poumian-Tapia and Ibarra-Obando (1999). In order to describe vegetative and reproductive phenology and the reproductive effort of Z. marina, we selected and sampled 4 locations monthly between June 1996 and November 1997. Locations were chosen to cover most of the bay area where Z. marina is present. At each location 6 sites were sampled, 3 in the subtidal environment $(-1.90,-0.50$, and $-0.10 \mathrm{~m}$ with respect to the mean lower low water, MLLW) and 3 in the intertidal environment $(+0.01,+0.10$, and $+0.20 \mathrm{~m}$ MLLW $)$. At each site, a 45-50 m transect was established to randomly collect three $0.04 \mathrm{~m}^{2}$ samples (24 quadrats per location per month), but given the ease of sampling at -0.50 and +0.10 , we duplicated transects and samples ( 96 quadrats per month).

Whole vegetative and reproductive shoots were collected within each quadrat, including rhizomes and roots. Collected material was sieved and rinsed with bay water to remove sediments. Shoots were placed in labeled plastic bags and transported to the lab in an ice chest. In the lab, both vegetative and reproductive shoots were rinsed with distilled water and treated separately. The material was immersed in $10 \%$ phosphoric acid for $5 \mathrm{~min}$ to separate epibionts and sediments. Dry weight was measured after drying the sample at $75{ }^{\circ} \mathrm{C}$ for $24 \mathrm{~h}$. Samples were separated into above- and belowground vegetative biomass. Vegetative shoot density was calculated as the number of shoots per square meter. The date of first reproductive shoot apparition, date of reproductive shoot release, and number of seeds per shoot were recorded. Reproductive shoots were separated into leaves and reproductive structures. The reproductive effort (RE) of $Z$. marina is the ratio of the dry weight of reproductive shoots to the total above- and below-ground vegetative biomass in dry weight multiplied by 100 , i.e., the proportion of total shoot biomass allocated to sexual reproduction. This method is common and reliable (Kaul 2016) for representing the proportion of plant energy invested in reproduction. Seeds are potential plants, so we used seed number and total biomass (vegetative and reproductive) to assess seed $\mathrm{RE}\left(\mathrm{RE}_{\mathrm{s}}\right)$, which
Nunca se han comparado simultáneamente la biomasa vegetativa, la biomasa sexual, la densidad y el tamaño de haces, y el esfuerzo reproductivo de $Z$. marina que crece en el gradiente de profundidad de la BSQ, desde el submareal somero hasta lo alto del intermareal, ni tampoco se han evaluado durante un evento ENOS. Sólo hay información escasa y fragmentada sobre la biomasa vegetativa y la fenología sexual asociada al esfuerzo reproductivo de Z. marina y cómo estas variables biológicas son afectadas por eventos inusuales de altas temperaturas, como las del ENOS, o en un gradiente de profundidad. Nuestra hipótesis es que el aumento de temperatura que ocurrió de 1996 al periodo ENOS de 1997-1998 resultó en una reducción de la biomasa vegetativa y un incremento de la fenología sexual de Z. marina. El mejor ambiente para la fenología reproductiva es el intermareal, mientras que el submareal representa un ambiente más propicio para el desarrollo de las características vegetativas.

Con la llegada del cambio climático necesitamos un conocimiento más profundo sobre el comportamiento de las praderas de Z. marina. Si los eventos de temperaturas extremas se hacen más frecuentes y el nivel del mar incrementa, debemos ser capaces de predecir cómo responderá la especie.

\section{MATERIALES Y MÉTODOS}

BSQ es una laguna costera $\left(30^{\circ} 30^{\prime} \mathrm{N}, 116^{\circ} 01^{\prime} \mathrm{W}\right)$ en la costa del Pacífico de Baja California, México. La ecología de su comunidad ha sido descrita por Poumian-Tapia e IbarraObando (1999). Para describir la fenología vegetativa y reproductiva y el esfuerzo reproductivo de $Z$. marina, seleccionamos 4 localidades y las muestreamos mensualmente de junio de 1996 a noviembre de 1997. Las localidades que se seleccionaron cubren gran parte del área de la bahía donde está presente Z. marina. En cada localidad se muestrearon 6 sitios, 3 en el submareal $(-1.90,-0.50$ y $-0.10 \mathrm{~m}$ respecto al nivel medio de la bajamar más baja [MLLW]) y 3 en el intermareal $(+0.01,+0.10 \mathrm{y}+0.20 \mathrm{~m}$ del MLLW $)$. En cada sitio se estableció un transecto de $45-50 \mathrm{~m}$ para recolectar al azar 3 muestras de $0.04 \mathrm{~m}^{2}$ (24 cuadrantes por localidad por mes), pero dada la facilidad de muestreo a -0.50 y +0.10 , duplicamos los transectos y las muestras ( 96 cuadrantes por mes).

En cada cuadrante se recolectó todo el material vegetativo y reproductivo, incluso los rizomas y las raíces. El material recolectado se tamizó y lavó con agua de la bahía para remover el sedimento. Los haces fueron colocados en bolsas de plástico etiquetadas y transportados al laboratorio en una hielera. En el laboratorio, tanto los haces vegetativos como los reproductivos, fueron lavados con agua destilada y tratados por separado. Las muestras se sumergieron en ácido fosfórico al $10 \%$ durante $5 \mathrm{~min}$ para separar los epibiontes y el sedimento. Determinamos el peso seco después de secar el material a $75^{\circ} \mathrm{C}$ durante $24 \mathrm{~h}$. La biomasa vegetativa se separó en biomasa foliar 
represents the proportion of total biomass invested in the production of seeds $\left(\mathrm{RE}_{\mathrm{s}}=\right.$ seeds $\cdot \mathrm{m}^{-2} /$ total biomass $\mathrm{g}$ dry wet $\left.[\mathrm{DW}] \cdot \mathrm{m}^{-2}\right)$.

We used air temperature at noon and at 3:00 AM (local time) and air humidity records from the Molino Viejo Tourist Hotel weather station. We also measured water temperature and photon irradiance at $-1.0 \mathrm{~m}$ with a Li-Cor Radiation Sensor (Lincoln, Nebraska, USA) using $19 \mathrm{~s}$ integration time; irradiance measurements at noon (local time) and all other measurements were taken until a consistent reading was obtained for $30 \mathrm{~s}$. Intertidal exposure time was calculated from the Centro de Investigación Científica y de Educación Superior de Ensenada tide charts (CICESE 2019).

Above- and below-ground vegetative biomass, shoot density, and shoot length data showed a normal distribution, so we used parametric statistics for their analyses (Zar 2010). Between-year comparisons were done over the same months: June-November 1996 and June-November 1997. Given the broad classification of our experimental plots (depth gradient from the subtidal to the intertidal zones, and between years) and to test whether the complete sets of homologous variables were significantly different, we applied parametric and non-parametric statistics, using the normal approximation where appropriate. In all cases, $\alpha=0.05$. The performed statistical tests, both Student's $t$ test and the parametric correlations, are summarized in Table 1.

\section{RESULTS}

Low water temperatures were recorded during winterspring, with the lowest $\left(16.9^{\circ} \mathrm{C}\right)$ in January 1996. Temperature increased during summer-autumn. The highest water temperature $\left(23.8^{\circ} \mathrm{C}\right)$ was recorded in August 1997. In 1997 water temperature was $1.5^{\circ} \mathrm{C}$ higher than in 1996, and it was higher than air temperature from January through April. During the same months, air temperature was $14.7^{\circ} \mathrm{C}$ higher in 1997 than in 1996. Mean air temperature was $5.3{ }^{\circ} \mathrm{C}$ higher in 1997 than in 1996. In February 1997 mean air temperature was $13.2^{\circ} \mathrm{C}$, and in August 1997 it reached $29.9^{\circ} \mathrm{C}$. The largest water-air temperature difference was $15.0^{\circ} \mathrm{C}$.

Photons showed no trend over time, nor were they correlated with other variables. They were higher in $1996\left(508.67 \pm 113.6 \mu \mathrm{E} \mathrm{m}^{-2} \cdot \mathrm{s}^{-1}\right)$ than in $1997(372.3 \pm$ $\left.62.9 \mu \mathrm{E} \mathrm{m} \mathrm{m}^{-2} \cdot \mathrm{s}^{-1}\right)$. Salinity did not show any noticeable effects on the biological variables. Exposure time for intertidal Z. marina beds ranged from 3.0 to $4.5 \mathrm{~h}$ during sunlight hours at low tides, for 10-19 days per month. We did not find a significant statistical effect of exposure time on any $Z$. marina biological variables in the intertidal zone. The results of the performed statistical tests are shown in Table 1.

Annual mean vegetative biomass $\left(\mathrm{g} \mathrm{DW} \cdot \mathrm{m}^{-2}\right)$ was significantly higher in the subtidal environment than in the intertidal environment, and it gradually decreased from -1.90 to $+0.20 \mathrm{~m}$ (Table 2). Vegetative biomass was higher by y biomasa subterránea. La densidad de haces vegetativos se calculó como el número de haces por metro cuadrado. También registramos la fecha de la primera aparición de haces reproductivos, la fecha del desprendimiento de los haces reproductivos maduros y el número de semillas por haz. Los haces reproductivos se separaron en hojas y estructuras reproductoras. El esfuerzo reproductivo (ER) de Z. marina se calculó como la razón entre el peso seco de los haces reproductivos y el total de la biomasa vegetativa foliar y subterránea en peso seco multiplicada por 100, i.e., la proporción de la biomasa total asignada a la reproducción sexual. Este método es muy común y confiable (Kaul 2016) para representar la proporción de energía que invierte la planta en la reproducción sexual. Siendo las semillas plantas potenciales, utilizamos el número total de semillas y la biomasa total (biomasa vegetativa y biomasa reproductiva) para estimar el ER invertido en las semillas $\left(E_{\mathrm{s}}\right)$, el cual representa la proporción de la biomasa total invertida en la producción de semillas $\left(\mathrm{ER}_{\mathrm{s}}=\right.$ semillas $\cdot \mathrm{m}^{-2} /$ biomasa total $\mathrm{g}$ peso seco $[\mathrm{PS}] \cdot \mathrm{m}^{-2}$ ).

Utilizamos los registros de la temperatura del aire al mediodía y a las 3:00 am (hora local) y la humedad relativa del aire tomados de la estación meteorológica del hotel Molino Viejo. También medimos la temperatura del agua y la irradiancia a $-1.0 \mathrm{~m}$ con un sensor de radiación Li-Cor (Lincoln, Nebraska, EUA) utilizando un tiempo de integración de $19 \mathrm{~s}$; la irradiancia al mediodía (horario local) y todas las mediciones se registraron hasta que se obtuvo una lectura constante durante $30 \mathrm{~s}$. El tiempo de exposición se calculó con las tablas de marea del Centro de Investigación Científica y de Educación Superior de Ensenada (CICESE 2019)

Los datos de biomasa vegetativa (foliar y subterránea), densidad de haces y longitud de haces mostraron una distribución normal, por lo que utilizamos análisis estadísticos paramétricos para su análisis (Zar 2010). Para las comparaciones entre años se utilizaron los mismos meses (junionoviembre de 1996 y junio-noviembre de 1997). Debido a la amplia clasificación de nuestros sitios experimentales (gradiente de profundidad del submareal al intermareal y entre años) y con la finalidad de probar si las series completas de las variables homólogas eran significativamente diferentes, aplicamos pruebas estadísticas paramétricas y no paramétricas, y en los casos requeridos, hicimos transformaciones para obtener normalidad. En todos los casos aplicamos $\alpha=$ 0.05. Las pruebas estadísticas realizadas, tanto las correlaciones paramétricas como la prueba de $t$ de Student, están resumidas en la Tabla 1.

\section{RESUltados}

Las temperaturas del agua bajas se registraron durante el invierno-primavera, con la más baja $\left(16.9{ }^{\circ} \mathrm{C}\right)$ en enero de 1996. La temperatura aumentó durante el verano-otoño. La temperatura del agua más alta $\left(23.8^{\circ} \mathrm{C}\right)$ se registró en agosto 
68.3\% in 1996 than in 1997 (Table 2). Above-ground biomass (leaves and sheaths) was higher than below-ground biomass (roots and rhizomes) at all subtidal sites year-round (Fig. 1). Subtidal below-ground biomass was lower than intertidal above-ground biomass from November through April. Above-ground vegetative biomass was significantly correlated with water temperature but not with belowground vegetative biomass. Almost all biological parameters were higher in 1996 than in 1997, especially above-ground biomass in the subtidal environment. Vegetative biomass was associated with water temperature from June 1996 to February 1997 and from February to November 1997.

Vegetative shoot density was higher in the intertidal environment than in the subtidal environment (Fig. 2). The highest vegetative shoot density was found at $+0.20 \mathrm{~m}$, decreasing at greater depths, with the lowest density at $-1.90 \mathrm{~m}$. Shoot density was highest at +0.01 and $+0.20 \mathrm{~m}$. In the subtidal environment, at $-0.20 \mathrm{~m}$, the highest shoot density was $1,460 \pm 286.5$ shoots $\cdot \mathrm{m}^{-2}$ and at the same depth the minimum was $472.1 \pm 85.77$ shoots $\cdot \mathrm{m}^{-2}$. Vegetative shoot density was not statistically different between years and was negatively associated with depth. It was also positively associated with vegetative biomass and with above- and below-ground biomass at almost all sites, but not with below-ground biomass at $-1.90 \mathrm{~m}$.

Mean vegetative shoot length (Fig. 3) was higher by more than $50 \%$ in the subtidal environment $(43.5 \pm 19.6 \mathrm{~cm})$ than in the intertidal environment $(20.9 \pm 12.4 \mathrm{~cm})$. Shoot length was highest $(87.2 \pm 38.7 \mathrm{~cm})$ at $-1.90 \mathrm{~m}$, and it decreased at shallower sites, with the lowest $(8.3 \pm 2.7 \mathrm{~cm})$ at $+0.20 \mathrm{~m}$. Vegetative shoot length in 1996 was statistically higher than in 1997, showed correlation with depth, and was significantly correlated with vegetative biomass.

Mean reproductive biomass was $9.2 \%$ of vegetative biomass $\left(11.3 \pm 3.2 \mathrm{~g} \mathrm{DW} \cdot \mathrm{m}^{-2}\right)$. Its proportion in 1997 was higher than in 1996 (Fig. 4). Mean reproductive biomass was higher in the intertidal zone than in the subtidal zone. During 1997 , in both the subtidal and intertidal zones, reproductive biomass peaked in July and was correlated with reproductive shoot density. Reproductive shoot density was lower in the subtidal environment than in the intertidal environment (Fig. 5). Peak reproductive shoot density values were different through time. Reproductive shoot density was higher in 1997 than in 1996. In May we found 25.0 15.2 reproductive shoots per square meter (RS $\cdot \mathrm{m}^{-2}$ ) in the subtidal zone and in August $118.8 \pm 24.4 \mathrm{RS} \cdot \mathrm{m}^{-2}$ in the intertidal zone. The lowest density $\left(16 \pm 1.2 \mathrm{RS} \cdot \mathrm{m}^{-2}\right)$ was found at $-1.90 \mathrm{~m}$, and the highest density in the intertidal environment (46.6 \pm $2.6 \mathrm{RS} \cdot \mathrm{m}^{-2}$ ) was found at $+0.20 \mathrm{~m}$. Reproductive shoot density correlated with depth and vegetative density.

Seed release took place from May to October in the subtidal environment and from May to November in the intertidal environment. Maximum mean values were $219.5 \pm$ 45.8 seeds per reproductive shoot at $-0.50 \mathrm{~m}$, reaching 238.2 seeds per reproductive shoot in the subtidal environment, de 1997. En 1997 la temperatura del agua fue $1.5^{\circ} \mathrm{C}$ más alta que en 1996 y la temperatura del agua fue más alta que la temperatura del aire de enero a abril. Durante los mismos meses, la temperatura del aire fue $14.7^{\circ} \mathrm{C}$ más alta en 1997 que en 1996. La temperatura promedio del aire fue $5.3{ }^{\circ} \mathrm{C}$ más alta en 1997 que en 1996. En febrero de 1997 la temperatura media del aire fue de $13.2{ }^{\circ} \mathrm{C}$ y en agosto de 1997 alcanzó $29.9^{\circ} \mathrm{C}$. La mayor diferencia de temperatura entre el agua y el aire fue de $15.0^{\circ} \mathrm{C}$.

La irradiancia no mostró tendencia alguna en el tiempo ni estuvo correlacionada con otras variables. Se registraron valores más altos en $1996\left(508.67 \pm 113.6 \mu \mathrm{Em}^{-2} \cdot \mathrm{s}^{-1}\right)$ que en $1997\left(372.3 \pm 62.9 \mu \mathrm{Em}^{-2} \cdot \mathrm{s}^{-1}\right)$. La salinidad no mostró efectos observables sobre las variables biológicas. Las mareas bajas se presentaron en las horas de sol durante 10-19 d por mes, y el tiempo de exposición al aire de las praderas de Z. marina en el intermareal varió de 3.0 a $4.5 \mathrm{~h}$ por día durante las mareas bajas. No encontramos ningún efecto estadístico significativo del tiempo de exposición en ninguna variable biológica de Z. marina en la zona intermareal. Los resultados de las pruebas estadísticas realizadas se muestran en la Tabla 1.

El promedio anual de la biomasa vegetativa $\left(\mathrm{g} \mathrm{PS} \cdot \mathrm{m}^{-2}\right.$ ) fue significativamente mayor en el submareal que en el intermareal, y disminuyó gradualmente de -1.90 a $+0.20 \mathrm{~m}$ (Tabla 2). La biomasa vegetativa fue mayor en un $68.3 \%$ en 1996 que en 1997 (Tabla 2). La biomasa foliar (hojas y vaina) fue mayor que la subterránea (raíces y rizomas) en todos los sitios del submareal y durante todo el año (Fig. 1). De noviembre a abril, la biomasa subterránea del submareal fue menor que la biomasa subterránea del intermareal. La biomasa vegetativa foliar estuvo correlacionada significativamente con la temperatura del agua, pero no con la biomasa subterránea. Casi todas las variables biológicas fueron más altas durante 1996 con respecto a 1997, especialmente la biomasa foliar del submareal. La biomasa vegetativa estuvo asociada con la temperatura del agua de junio de 1996 a febrero de 1997 y de febrero a noviembre de 1997.

La densidad de los haces vegetativos fue mayor en el intermareal que en el submareal (Fig. 2). El valor más alto de densidad de haces vegetativos se encontró a $+0.20 \mathrm{~m}$ y disminuyó hacia sitios más profundos, con la menor densidad de haces a $-1.90 \mathrm{~m}$. En el ambiente submareal, a $-0.20 \mathrm{~m}$, la densidad más alta de haces fue de 1,460 \pm 286.5 haces $\cdot \mathrm{m}^{-2}$ y la más baja fue de $472.1 \pm 85.77$ haces $\cdot \mathrm{m}^{-2}$. La densidad de haces vegetativos no mostró diferencias estadísticamente significativas entre años y estuvo asociada negativamente con la profundidad. También se asoció positivamente con la biomasa vegetativa y con la biomasa foliar y subterránea en casi todos los sitios, pero no con la biomasa subterránea a $-1.90 \mathrm{~m}$.

La longitud media de los haces vegetativos (Fig. 3) fue superior en más del $50 \%$ en el submareal $(43.5 \pm 19.6 \mathrm{~cm})$ que en el intermareal $(20.9 \pm 12.4 \mathrm{~cm})$. La longitud de haces fue mayor $(87.2 \pm 38.7 \mathrm{~cm})$ a $-1.90 \mathrm{~m}$ y disminuyó en sitios 
and $151.3 \pm 21.5$ seeds per reproductive shoot at $+0.10 \mathrm{~m}$, reaching 185.4 seeds per reproductive shoot in the intertidal environment, both in July. In 1997, the mean number of seeds per reproductive shoot in the subtidal and the intertidal environments was, respectively, 138 and 73 seeds $\cdot \mathrm{m}^{-2}$ (Table 3), and it correlated with reproductive biomass.

RE was higher in 1997 than in 1996. The June 1997 values were higher than those for June 1996 and they were higher in the intertidal environment. Mean RE using seed number was $22.6 \pm 12.1$ seeds $\mathrm{g} \mathrm{DW} \cdot \mathrm{m}^{-2}$. $\mathrm{RE}_{\mathrm{s}}$ was lower in 1996 than in 1997. In the subtidal environment mean $R_{s}$ decreased from May to October. In the intertidal environment $\mathrm{RE}_{\mathrm{s}}$ was $61.5 \pm 23.1$ seeds $\mathrm{g} \mathrm{DW} \cdot \mathrm{m}^{-2}$. $\mathrm{RE}_{\mathrm{s}}$ started in May $\left(36.8 \pm 0.6\right.$ seeds $\left.\mathrm{g} \mathrm{DW} \cdot \mathrm{m}^{-2}\right)$ and ended in November $\left(3.4 \pm 0.2\right.$ seeds $\left.\mathrm{g} \mathrm{DW} \cdot \mathrm{m}^{-2}\right)$. We found maximum values for this variable in the intertidal environment in June, which coincided with maximum reproductive biomass (Fig. 3), one month earlier than the peak for vegetative biomass. In both years RE was lower at $-1.90 \mathrm{~m}$ in the subtidal environment más someros, con la menor longitud de los haces $(8.3 \pm$ $2.7 \mathrm{~cm}$ ) a $+0.20 \mathrm{~m}$. La longitud de los haces vegetativos en 1996 fue estadísticamente mayor que en 1997, se correlacionó con la profundidad y se correlacionó significativamente con la biomasa vegetativa.

El promedio de la biomasa reproductiva (11.3 \pm $3.2 \mathrm{~g} \mathrm{PS} \cdot \mathrm{m}^{-2}$ ) fue $9.2 \%$ de la biomasa vegetativa. Su proporción en 1997 fue mayor que en 1996 (Fig. 4). La biomasa reproductiva media del intermareal fue mayor que la del submareal. Durante 1997, tanto en el submareal como en el intermareal, la biomasa reproductiva alcanzó su punto máximo en julio y se correlacionó con la densidad de haces reproductivos. La densidad de haces reproductivos fue menor en el submareal que en el intermareal (Fig. 5). Los valores máximos de la densidad de haces reproductivos fueron diferentes a través del tiempo. La densidad de haces reproductivos fue mayor durante 1997 que en 1996. Encontramos $25.0 \pm 15.2$ haces reproductivos por metro cuadrado $\left(\mathrm{HR} \cdot \mathrm{m}^{-2}\right)$ en el submareal en mayo y $118.8 \pm 24.4 \mathrm{HR} \cdot \mathrm{m}^{-2}$

Table 1. Statistical tests performed on data for Zostera marina vegetative and reproductive biomass in San Quintín Bay, Baja California, Mexico. Sample size $=n$.

Tabla 1. Pruebas estadísticas realizadas con los datos de la biomasa vegetativa y reproductiva de Zostera marina en la bahía San Quintín, Baja California, México. $n=$ tamaño de muestra.

\begin{tabular}{|c|c|c|c|c|}
\hline Statistical test & Variables & $n$ & Test value & $P$ value \\
\hline \multirow[t]{3}{*}{ Student's $t$} & Annual vegetative biomass (1996 vs 1997) & 18 & 8.43 & $<0.05$ \\
\hline & $\begin{array}{l}\text { Above and below ground vegetative biomass at the } \\
\text { subtidal (1996 vs 1997) }\end{array}$ & 18 & 10.72 & $<0.05$ \\
\hline & Vegetative shoot density intertidal $v s$ subtidal & 18 & 7.56 & $<0.05$ \\
\hline \multirow[t]{15}{*}{ Correlation $(r)$} & Vegetative shoot length intertidal vs subtidal & 18 & 9.23 & $<0.05$ \\
\hline & Vegetative biomass $v s$ depth & 6 & 0.74 & $<0.05$ \\
\hline & $\begin{array}{l}\text { Above ground vegetative biomass at the subtidal } v s \\
\text { below ground }\end{array}$ & 18 & 0.84 & $<0.05$ \\
\hline & $\begin{array}{l}\text { June-Feb vegetative biomass at the subtidal } v s \\
\text { temperature (1996) }\end{array}$ & 9 & 0.74 & $<0.05$ \\
\hline & $\begin{array}{l}\text { Feb-Nov vegetative biomass at the subtidal } v s \\
\text { temperature (1997) }\end{array}$ & 10 & 0.78 & $<0.05$ \\
\hline & Vegetative shoot density $v s$ depth & 6 & -0.50 & $<0.05$ \\
\hline & Vegetative shoot density $v s$ vegetative biomass & 18 & 0.57 & $<0.05$ \\
\hline & Vegetative shoot length $v s$ depth & 6 & 0.94 & $<0.05$ \\
\hline & Vegetative shoot length $v s$ vegetative biomass & 18 & 0.72 & $<0.05$ \\
\hline & $\begin{array}{l}\text { Subtidal and intertidal reproductive biomass vs re- } \\
\text { productive shoot density (1996 and1997) }\end{array}$ & 15 & 0.81 & $<0.05$ \\
\hline & Reproductive shoot density $v s$ depth & 6 & 0.69 & $<0.05$ \\
\hline & Reproductive shoot density $v s$ vegetative density & 15 & 0.59 & $<0.05$ \\
\hline & Seeds/reproductive shoot $v s$ reproductive biomass & 10 & 0.69 & $>0.05$ \\
\hline & Reproductive effort $v s$ vegetative density & 15 & 0.62 & $<0.05$ \\
\hline & Reproductive effort $v s$ reproductive biomass & 15 & 0.72 & $<0.05$ \\
\hline
\end{tabular}


Table 2. Mean vegetative biomass values $\left(\mathrm{g} \mathrm{DW} \cdot \mathrm{m}^{-2}\right.$ ) for shoots (above-ground biomass) and rhizomes/roots (below-ground biomass) at each site sampled in the subtidal and intertidal environments. Mean values are also shown per year $(1996,1997)$ for above- and below-ground vegetative biomass and per row.

Tabla 2. Valores de la media de haces (biomasa foliar) y rizomas y raíces (biomasa subterránea) en cada sitio muestreado en el submareal y el intermareal $\left(\mathrm{g}\right.$ PS $\left.\cdot \mathrm{m}^{-2}\right)$. También se muestran las medias de biomasa vegetativa foliar y subterránea por año (1996 y 1997) y por fila.

\begin{tabular}{|c|c|c|c|c|c|c|c|}
\hline & \multicolumn{3}{|c|}{ Subtidal } & \multicolumn{3}{|c|}{ Intertidal } & \multirow[b]{2}{*}{ Mean } \\
\hline & $-1.9 \mathrm{~m}$ & $-0.50 \mathrm{~m}$ & $-0.10 \mathrm{~m}$ & $+0.01 \mathrm{~m}$ & $+0.10 \mathrm{~m}$ & $+0.20 \mathrm{~m}$ & \\
\hline Above & 303.3 & 227.5 & 230.8 & 152.9 & 113.9 & 74.2 & 183.8 \\
\hline Below & 99.6 & 71.8 & 68.4 & 57.2 & 42.8 & 35.1 & 62.5 \\
\hline 1996 & 232.5 & 201.9 & 172.4 & 119.7 & 93.9 & 70.2 & 148.4 \\
\hline 1997 & 171.1 & 102.3 & 125.9 & 89.9 & 63.7 & 41.1 & 99.0 \\
\hline
\end{tabular}

$\left(6.2 \pm 0.5\right.$ seeds $\left.\mathrm{g} \mathrm{DW} \cdot \mathrm{m}^{-2}\right)$, while the highest in the intertidal environment was found at $+0.10 \mathrm{~m}(25.5 \pm 0.3$ seeds $\mathrm{g} \mathrm{DW} \cdot \mathrm{m}^{-2}$ ). Also RE values were higher at both depths and all sites in 1997 than in 1996 (Table 3). RE was positively correlated with vegetative density and reproductive biomass.

\section{Discussion}

SQB is the southern range limit for Z. marina and other land and marine organisms in the Northern Hemisphere (Cabello-Pasini et al. 2002). It is located in a temperate zone limit and close to a desert; therefore, dramatic changes in air temperature are common. SQB was impacted by the 1997-1998 ENSO event, the most drastic ENSO event recorded in modern history (Kumar et al. 2001). We recorded a $1.5^{\circ} \mathrm{C}$ increase in sea surface temperature in 1997 in SQB with respect to 1996. This increase is higher than the $0.25{ }^{\circ} \mathrm{C}$ increase measured during other ENSO events (Trenberth et al. 2002). This extraordinary increase was critical for Z. marina, as shown by the low above-ground biomass produced and by the below-ground biomass at a smaller scale. The same trend was reported by Johnson et al. (2003) for San Diego, California, USA, about $280 \mathrm{~km}$ due north, and by Shields et al. (2018) for Chesapeake Bay, Virginia. This decrease in biomass production associated with the increase in water temperature is evidence that $Z$. marina underwent continuous stress during the ENSO event. We confirmed our hypothesis that during our study period, the increase in surface water temperature caused some of the productivity and phenological differences between years. In June, at low tides, intertidal eelgrass beds in SQB were exposed to higher air temperatures $\left(39.9^{\circ} \mathrm{C}\right)$ in 1997 than in $1996\left(20.1^{\circ} \mathrm{C}\right)$. Minimum temperatures in November were $3.9{ }^{\circ} \mathrm{C}$ and $12.8{ }^{\circ} \mathrm{C}$ for 1996 and 1997, respectively. In SQB Cabello-Pasini et al. (2003) recorded $22.0^{\circ} \mathrm{C}$ as maximum mean air and water temperatures during 1999-2000, but we recorded $28.5^{\circ} \mathrm{C}$ en el intermareal en agosto. La densidad más baja (16 \pm $1.2 \mathrm{HR} \cdot \mathrm{m}^{-2}$ ) se encontró a $-1.90 \mathrm{~m}$ y la densidad más alta $\left(46.6 \pm 2.6 \mathrm{HR} \cdot \mathrm{m}^{-2}\right)$ en el intermareal se encontró a $+0.20 \mathrm{~m}$. La densidad de haces reproductivos se correlacionó con la profundidad y la densidad vegetativa.

El desprendimiento de semillas ocurrió de mayo a octubre en el submareal y de mayo a noviembre en el intermareal. Los promedios máximos registrados fueron $219.5 \pm 45.8$ semillas por haz reproductivo a $-0.50 \mathrm{~m}$, con un máximo de 238.2 semillas por haz reproductivo en el submareal, y $151.3 \pm 21.5$ semillas por haz reproductivo a $+0.10 \mathrm{~m}$, con un máximo de 185.4 semillas por haz en el intermareal, ambos casos en julio. Durante 1997, la media de semillas por haz reproductor en el submareal y el intermareal fue, respectivamente, 138 y 73 semillas $\cdot \mathrm{m}^{-2}$ (Tabla 3 ) y estuvo correlacionada con la biomasa reproductiva.

El ER fue mayor en 1997 que en 1996. Los valores de junio de 1997 fueron más altos que los de junio de 1996 y los valores más altos se presentaron en el intermareal. La media de ER, utilizando el número de semillas, fue $22.6 \pm 12.1 \mathrm{~g}$ PS semillas $\cdot \mathrm{m}^{-2}$. El ER fue menor en 1996 que en 1997. En el submareal, la media del $E_{\mathrm{s}}$ disminuyó de mayo a octubre. En el intermareal, el $E_{\mathrm{s}}$ fue de $61.5 \pm 23.1 \mathrm{~g}$ PS semillas $\cdot \mathrm{m}^{-2}$. El $\mathrm{ER}_{\mathrm{s}}$ inició en mayo $\left(36.8 \pm 0.6 \mathrm{~g}\right.$ PS semillas $\left.\cdot \mathrm{m}^{-2}\right)$ y finalizó en noviembre (3.4 \pm $0.2 \mathrm{~g}$ PS semillas $\cdot \mathrm{m}^{-2}$ ). Encontramos valores máximos de esta variable en junio en el intermareal, lo cual coincidió con el pico de biomasa reproductiva (Fig. 3), un mes antes que el pico máximo de biomasa vegetativa. En ambos años el menor valor $\left(6.2 \pm 0.5 \mathrm{~g}\right.$ PS semillas $\left.\cdot \mathrm{m}^{-2}\right)$ de ER se presentó en el submareal a $-1.90 \mathrm{~m}$, mientras que el valor máximo $\left(25.5 \pm 0.3 \mathrm{~g}\right.$ PS semillas $\left.\cdot \mathrm{m}^{-2}\right)$ se registró en el intermareal a $+0.10 \mathrm{~m}$. Además, se encontraron mayores valores de ER en todos los sitios del submareal y del intermareal durante 1997 que durante 1996 (Tabla 3). El ER estuvo significativamente correlacionado con la biomasa reproductiva y con la densidad de haces vegetativos. 
for air temperature and $23.8{ }^{\circ} \mathrm{C}$ for water temperature. Mean temperature was $2.4{ }^{\circ} \mathrm{C}$ warmer in air than in water during 1997. Similar water temperature values were found by Hernández-Carmona et al. (2011) on the central Baja California coast, $300 \mathrm{~km}$ south of SQB.

The only variables that showed no differences between years were vegetative and reproductive densities. All the other variables measured for Z. marina showed significant differences as a function of depth, site, time, and year (Tables 2, 3). Differences were notable for vegetative aboveground biomass and very evident for reproductive phenology. Differences between subtidal and intertidal depths were evident, but they were more site-specific, especially in both subtidal and intertidal boundaries. The intertidal environment had lower vegetative and reproductive biomass, shorter vegetative and reproductive shoots, higher vegetative and reproductive shoot density, and a longer reproductive cycle.

\section{DiscUSIÓN}

La BSQ está en el límite sur de la distribución de $Z$. marina y de otros organismos terrestres y marinos en el hemisferio norte (Cabello-Pasini et al. 2002). La bahía está ubicada en el límite de la zona templada y cerca de un desierto, por lo que los cambios drásticos en la temperatura del aire son comunes. La BSQ estuvo sujeta al evento ENOS de 1997-1998, el evento ENOS más drástico en la historia reciente (Kumar et al. 2001). En este trabajo registramos un aumento de $1.5^{\circ} \mathrm{C}$ en la temperatura superficial del mar en BSQ en 1997 con respecto a 1996. Este aumento es mayor que el aumento de $0.25^{\circ} \mathrm{C}$ medido en otros eventos ENOS (Trenberth et al. 2002). Este aumento extraordinario fue crítico para Z. marina, como lo demuestra la baja producción de biomasa foliar $y$, en menor escala, la biomasa subterránea. La misma tendencia fue reportada por Johnson et al.
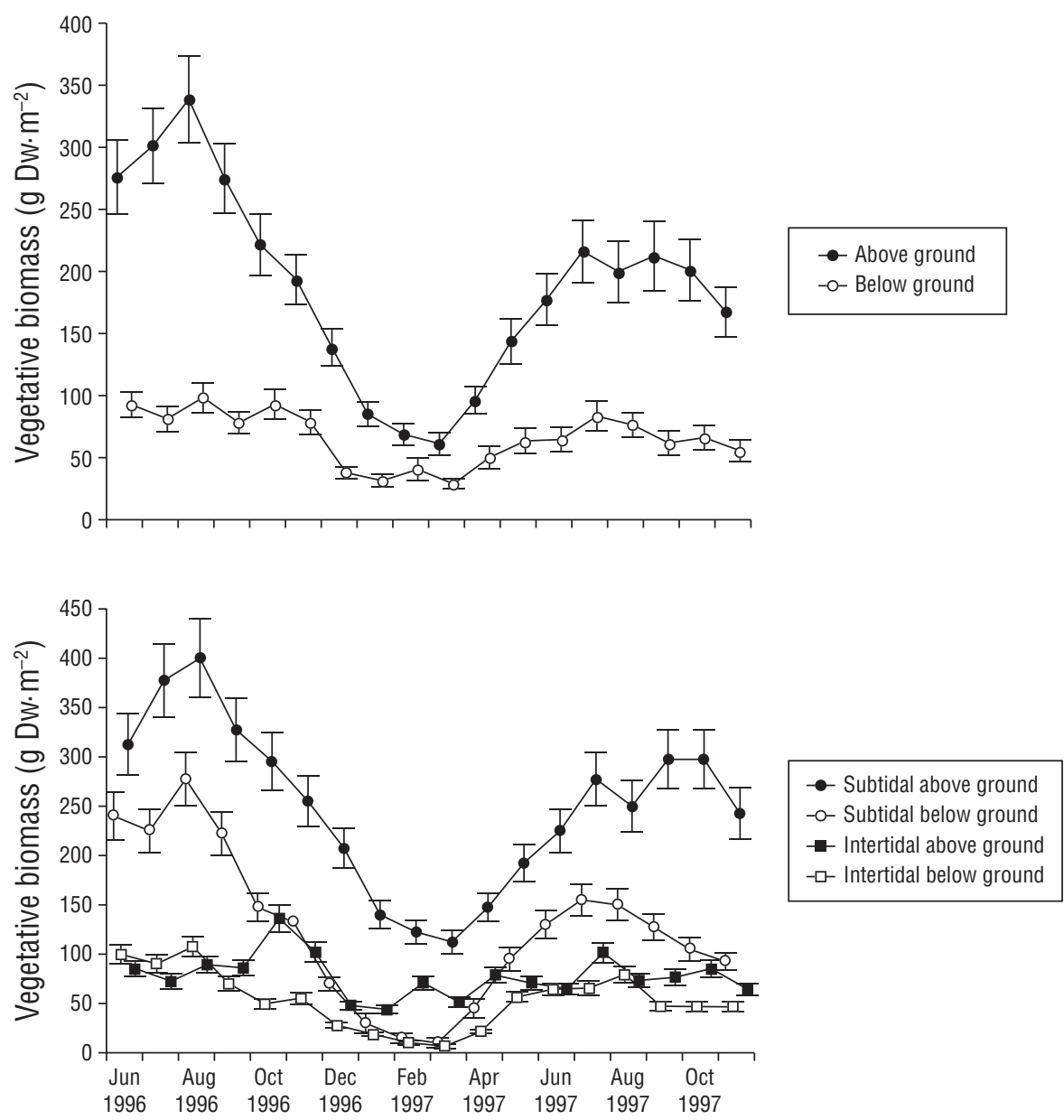

Figure 1. Annual above- and below-ground biomass trends (upper panel) and annual above- and below-ground vegetative biomass trends (lower panel) for Zostera marina in subtidal and intertidal environments in San Quintín Bay between June 1996 and October 1997. Bars in both panels are standard errors.

Figura 1. Tendencias anuales de la biomasa foliar y subterránea (panel superior) y tendencias anuales de la biomasa vegetativa foliar y subterránea en los ambientes submareal e intermareal (panel inferior) en la bahía San Quintín entre junio de 1996 y octubre de 1997. En ambos paneles, las barras representan el error estándar. 


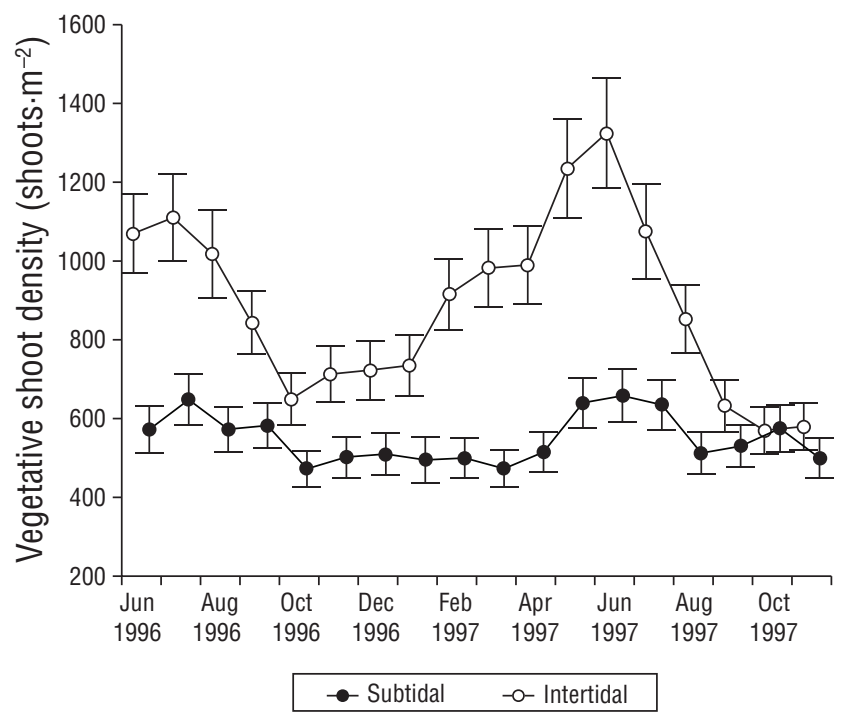

Figure 2. Annual trends of vegetative shoot density in subtidal and intertidal environments in San Quintín Bay between June 1996 and October 1997. Bars are standard errors.

Figura 2. Tendencias anuales de la densidad de haces vegetativos en los ambientes submareal e intermareal en la bahía San Quintín entre junio de 1996 y octubre de 1997. Las barras representan el error estándar.

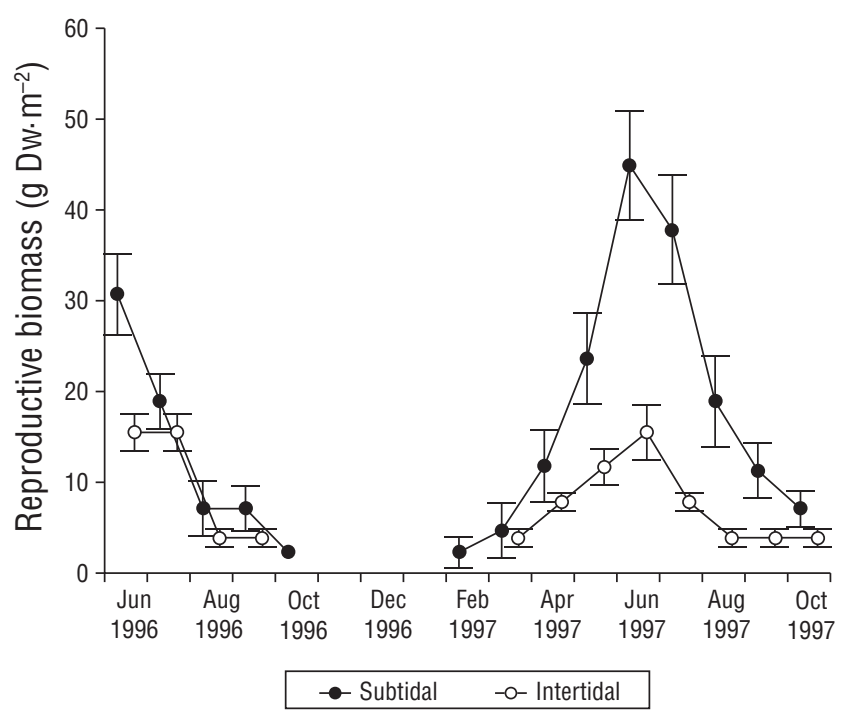

Figure 4. Reproductive Zostera marina biomass in subtidal and intertidal environments in San Quintín Bay between June 1996 and October 1997. Bars are standard errors.

Figura 4. Biomasa reproductiva de Zostera marina en las zonas intermareal y submareal de la bahía San Quintín entre junio de 1996 y octubre de 1997. Las barras representan el error estándar.

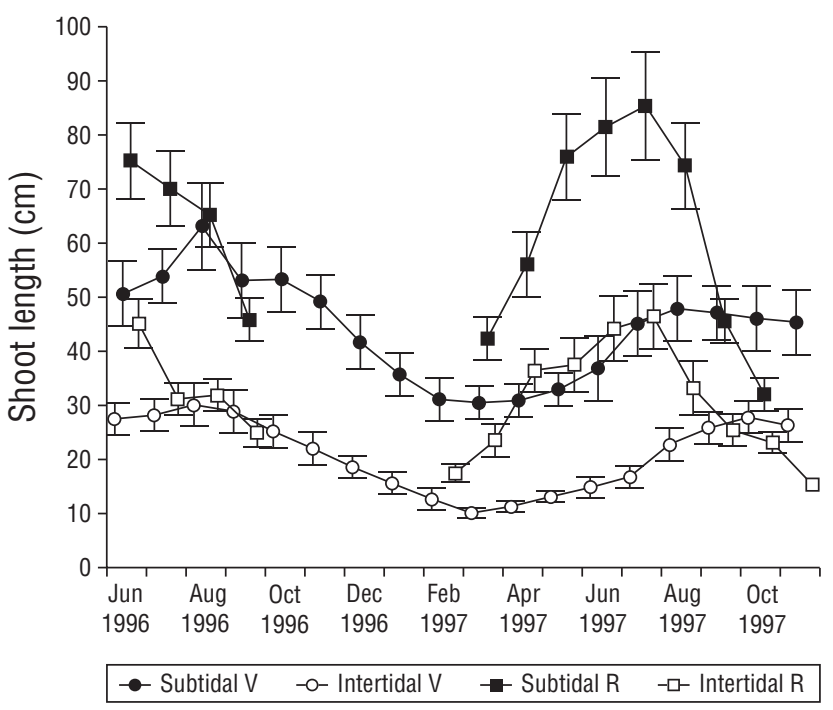

Figure 3. Annual trends of vegetative shoot length in subtidal and intertidal environments in San Quintín Bay between June 1996 and October 1997. Bars are standard errors. V, vegetative; R, reproductive.

Figura3. Tendencias anuales de la longitud de los haces vegetativos en los ambientes submareal e intermareal en la bahía San Quintín entre junio de 1996 y octubre de 1997 . V, vegetativo; $R=$ reproductivo. Las barras representan el error estándar.

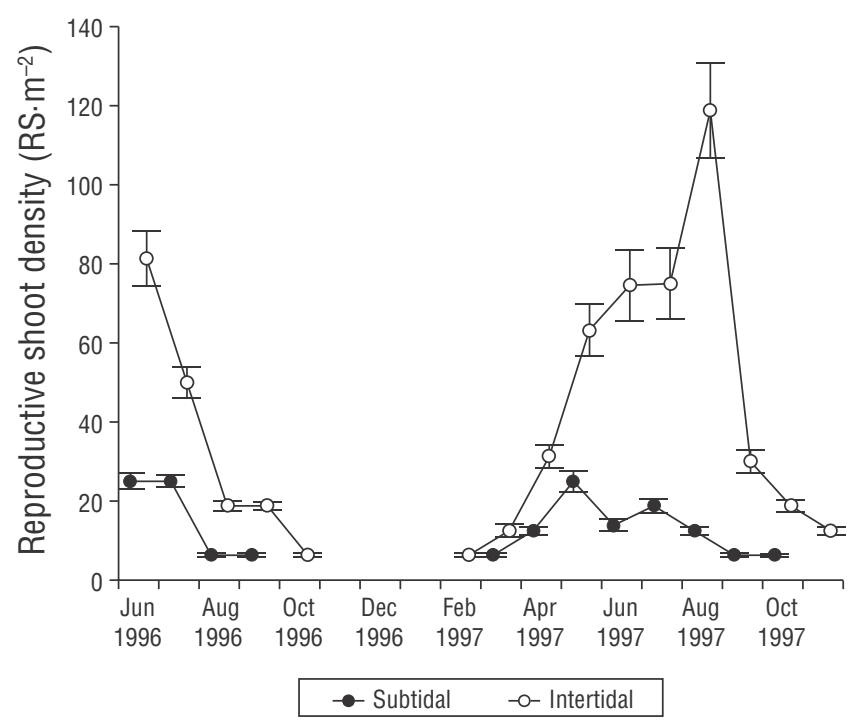

Figure 5. Reproductive Zostera marina shoot density in subtidal and intertidal environments in San Quintín Bay between June 1996 and October 1997. Bars are standard errors. RS $=$ reproductive shoot.

Figura 5. Densidad de haces reproductivos de Zostera marina en las zonas intermareal y submareal de la bahía San Quintín entre junio de 1996 y octubre de 1997. Las barras representan el error estándar. $\mathrm{RS}=$ haz reproductivo. 
Table 3. Structural features of Zostera marina reproductive shoots from both the subtidal and the intertidal environments in San Quintín Bay, Baja California, Mexico, for the years 1996 and 1997. Values show the mean (standard error) for the 4 sampled sites. SM $=$ seeds $\cdot \mathrm{m}^{-2} ; \mathrm{RE}=$ reproductive effort $\left(\right.$ seeds $\left.\mathrm{g} \mathrm{DW} \cdot \mathrm{m}^{-2}\right) ; \mathrm{SG}=$ seeds per reproductive shoot; $\mathrm{SW}=$ shoot weight $\left(\mathrm{g} \mathrm{DW} \cdot\right.$ shoot $\left.^{-1}\right)$.

Tabla 3. Características estructurales de los haces reproductivos de Zostera marina de los ambientes submareal e intermareal en la bahía San Quintín, Baja California, México, durante 1996 y 1997. Los valores representan la media (error estándar) para los 4 sitios muestreados. $\mathrm{SM}=$ semillas $\cdot \mathrm{m}^{-2} ; \mathrm{RE}=$ esfuerzo reproductivo (semillas $\mathrm{g} \mathrm{PS} \cdot \mathrm{m}^{-2}$ ); $\mathrm{SG}=$ semillas por haz reproductivo; $\mathrm{SW}=$ peso del haz $\left(\mathrm{g}\right.$ PS $\left.\cdot \mathrm{haz}^{-1}\right)$.

\begin{tabular}{lcccc}
\hline Environment & SM & RE & SG & SW \\
\hline Subtidal 1996 & $1,659(125.0)$ & $3.4(0.8)$ & $96(8.6)$ & $0.71(0.01)$ \\
Intertidal 1996 & $3,607(295.4)$ & $10.4(1.2)$ & $101(11.6)$ & $0.42(0.01)$ \\
Subtidal 1997 & $1,967(23.4)$ & $6.2(0.5)$ & $138(11.5)$ & $0.62(0.01)$ \\
Intertidal 1997 & $4,293(489.9)$ & $25.5(0.3)$ & $73(6.6)$ & $0.38(0.01)$ \\
Subtidal 1996 & $1,659(125.0)$ & $3.4(0.8)$ & $96(8.6)$ & $0.71(0.01)$ \\
\hline
\end{tabular}

Although RE decreased at the deepest site $(-1.90 \mathrm{~m})$, the best $Z$. marina stands were found between -0.50 and $+0.10 \mathrm{~m}$. This distribution of reproductive shoot density suggests that $Z$. marina is more parsimonious in the area that overlaps the subtidal and intertidal environments, and its distributional edges are well adapted to colonize new spaces. Both extremes in the depth range, -1.90 and $+0.20 \mathrm{~m}$, in SQB act as shoot expansion limits for Z. marina.

Edge theory states that organisms in edge populations face harsher conditions, whereas populations that survive and reproduce in the distributional boundary may disperse a greater number of seeds than those entering the boundary from outside (Cadenasso et al. 2003). In SQB the deepest edge acts as a control for the reproductive shoot, and the highest edge as a control for vegetative biomass. We found reduced $\mathrm{RE}$ in a small number of reproductive shoots at $-1.90 \mathrm{~m}$, the deepest site. We also recorded low vegetative biomass at $+0.20 \mathrm{~m}$ (Table 2). Light is probably limiting the distribution of $Z$. marina at deeper sites (Cabello-Pasini et al. 2002, Bertelli and Unsworth 2018), and desiccation is limiting its expansion to elevations above the tideline with longer air exposure (Nielsen et al. 2002). Zostera marina confronts these conditions with high vegetative biomass and shoot density. Edge populations may experience greater variability in survival and reproduction because they experience both harsh and limiting factors more frequently (Sexton et al. 2009). Here, sexual reproduction plays an important role in the species' adaptation to a constantly changing environment or in the invasion of new spaces. Given the unknown and largely unpredictable changes in the environment that will be produced by climate change, the chances of survival for $Z$. marina can change drastically at its southernmost range.

The responses of reproductive shoot density to light gradients found in our study are similar to those reported for perennial populations of $Z$. marina and other seagrass species (Henderson and Hacker 2015). Lee et al. (2005) suggested
(2003) para San Diego, California, EUA, aproximadamente $280 \mathrm{~km}$ al norte, y por Shields et al. (2018) para la bahía de Chesapeake, Virginia. Esta disminución en la producción de biomasa asociada con un incremento en la temperatura es evidencia de que $Z$. marina estuvo bajo estrés continuo durante el evento ENOS. Confirmamos nuestra hipótesis de que durante el periodo de estudio, el aumento de la temperatura del agua superficial causó algunas de las diferencias fenológicas y de productividad entre los años de muestreo. Durante las mareas bajas de junio de 1997, las praderas del pasto marino del intermareal en BSQ estuvieron expuestas a temperaturas del aire más altas $\left(39.9^{\circ} \mathrm{C}\right)$ que en 1996 $\left(20.1^{\circ} \mathrm{C}\right)$. Las temperaturas mínimas en noviembre fueron 3.9 y $12.8^{\circ} \mathrm{C}$ para 1996 y 1997 , respectivamente. Cabello-Pasini et al. (2003) registraron $22.0^{\circ} \mathrm{C}$ como promedio máximo de la temperatura del aire y del agua durante 1999-2000, mientras que nosotros registramos $28.5^{\circ} \mathrm{C}$ en el aire y $23.8^{\circ} \mathrm{C}$ en el agua. La temperatura media fue $2.4^{\circ} \mathrm{C}$ más cálida en el aire que en el agua durante 1997. Hernández-Carmona et al. (2011) encontraron temperaturas del agua similares en las costas centrales de Baja California, $300 \mathrm{~km}$ al sur de BSQ.

Las únicas variables que no mostraron diferencias entre años fueron las densidades vegetativas y reproductivas. Todas las demás variables medidas para $Z$. marina mostraron diferencias significativas en función de la profundidad, el sitio, el tiempo y los años (Tablas 2, 3). Las diferencias fueron notables en la biomasa vegetativa foliar y muy evidentes en la fenología reproductiva. Las diferencias entre las profundidades del submareal y el intermareal fueron evidentes, pero fueron muy específicas a cada sitio, especialmente en los límites del submareal y el intermareal. El ambiente intermareal tuvo menor biomasa vegetativa y reproductiva, haces vegetativos y reproductivos más cortos, mayor densidad de haces vegetativos y reproductivos, y un ciclo reproductivo más largo.

Aunque el ER disminuyó en el sitio más profundo $(-1.90 \mathrm{~m})$, los mejores sitios para Z. marina estuvieron entre 
that increases in water temperature trigger RE. Qin et al. (2014) found that in response to subtidal disturbances and stress, Z. marina increased seed production and RE. This trend has been described for other seagrasses (Cabaço and Santos 2012) but was not the case for Z. marina at $-1.90 \mathrm{~m}$ in SQB.

Vegetative and reproductive shoot lengths and densities were not significantly different between years. Vegetative and reproductive shoot lengths were not affected by temperature, but they were significantly associated with depth. Longer shoots were found at deeper sites, but the effect was negative on shoot density; Nielsen et al. (2002) and Henderson and Hacker (2015) reported similar results. The length of the reproductive cycle and the timing of reproductive shoots and their release vary with light or temperature (Johnson et al. 2003, Lee et al. 2005). We found differences in biomass but not in density between years. Similar patterns were described by Olesen and Sand-Jensen (1994) for eelgrass populations subject to severe biomass reductions, where biomass was predominantly allocated to extensive shoot recruitment.

Climate change is shifting the ranges of taxa, communities, and ecosystems, and the dynamics of populations that inhabit the latitudinal margins of the ranges will be critically important in determining species responses to climate change (Hampe and Petit 2005). SQB is a lagoon with low incident radiation and high turbidity (Nielsen et al. 2002, Cabello-Pasini et al. 2003), where the seasonal temperature trend has an important effect on biomass and growth (Ibarra-Obando et al. 1997, Poumian-Tapia and IbarraObando 1999, Cabello-Pasini et al. 2003). We observed the same trend in our study, high biomass in summer and low in winter (Figs. 1-3). We found that above-ground vegetative biomass increased by more than 4-fold in 1996-1997 $\left(187.5 \pm 79.7 \mathrm{~g} \mathrm{DW} \cdot \mathrm{m}^{-2}\right)$ compared with that during the 1999 2000 normal conditions $\left(40.0 \pm 1.6 \mathrm{~g} \mathrm{DW} \cdot \mathrm{m}^{-2}\right)($ CabelloPasini et al. 2003) and the 1992-1993 ENSO event $\left(57.02 \mathrm{~g} \mathrm{DW} \cdot \mathrm{m}^{-2}\right)$ (Echavarria-Heras et al. 2006). Most of these differences could be attributed to ENSO effects, as shown by the low biomass values during the 1986-1987 ENSO (Ibarra-Obando et al. 1997). The low biomass reported by Ibarra-Obando et al. (1997) during the 1986-1987 ENSO is underestimated because they only analyzed leaf biomass at the intertidal level. Intertidal shoot density in our study was $889.6 \pm 152.7$ shoots $\cdot \mathrm{m}^{-2}$, which is similar to the 400 1,390 shoots $\cdot \mathrm{m}^{-2}$ found during the 1991-1992 ENSO event by Echavarria-Heras et al. (2006) but slightly different from the $695 \pm 54$ shoots $\cdot \mathrm{m}^{-2}$ found during 1999-2000 (nonENSO years) by Cabello-Pasini et al. (2003). These studies show that shoot density was not significantly affected by ENSO events.

Reproductive strategies can result from optimal resource allocation between vegetative growth and sexual reproduction (Freitas-Coelho et al. 2005), with a balance being established between vegetative and reproductive biomass to fit
$-0.50 \mathrm{y}+0.10 \mathrm{~m}$. Esta distribución de la densidad de haces reproductivos sugiere que la especie es más parsimoniosa en el área donde se juntan el submareal y el intermareal y que los haces de $Z$. marina que se localizan en los bordes de las praderas están bien adaptados para colonizar nuevos espacios. Para Z. marina, ambos extremos del intervalo de profundidad, $-1.90 \mathrm{y}+0.20 \mathrm{~m}$, en BSQ actúan como límites de expansión de la pradera.

La teoría del borde establece que los organismos en las poblaciones de borde enfrentan condiciones muy adversas, mientras que las poblaciones que sobreviven y se reproducen en los límites de su distribución pueden dispersar una mayor cantidad de semillas que las que llegan del exterior (Cadenasso et al. 2003). En BSQ el borde más profundo actúa como control de los haces reproductivos y el más somero como control de la biomasa vegetativa. Encontramos menos ER en una cantidad baja de haces reproductivos a $-1.90 \mathrm{~m}$, el sitio más profundo. También registramos una baja biomasa de haces vegetativos a $+0.20 \mathrm{~m}$ (Tabla 2 ). La luz probablemente está limitando la distribución de Z. marina en los sitios más profundos (Cabello-Pasini et al. 2002, Bertelli y Unsworth 2018), y la desecación está limitando su expansión hacia elevaciones por encima de la línea de mareas, con tiempos de exposición al aire más prolongados (Nielsen et al. 2002). Zostera marina enfrenta estas condiciones produciendo gran cantidad de biomasa vegetativa y una alta densidad de haces. Las poblaciones de borde pueden experimentar mayor variabilidad en la supervivencia y la reproducción porque están sujetas, con mayor frecuencia, a presiones severas y factores limitantes (Sexton et al. 2009). En estos límites de distribución de las poblaciones, la reproducción sexual es un proceso importante en la adaptación de la especie a un entorno en constante cambio o en el proceso de invasión de nuevos espacios. Debido a los cambios desconocidos y en gran parte impredecibles en el medio ambiente que serán producidos por el cambio climático, las posibilidades de supervivencia de $Z$. marina pueden cambiar drásticamente en su intervalo de distribución más sureña.

Las respuestas de la densidad de haces reproductivos a los gradientes de luz encontradas en este estudio son similares a las que se han reportado para poblaciones perenes de Z. marina y otras especies de pastos marinos (Henderson y Hacker 2015). Lee et al. (2005) sugirieron que los incrementos en la temperatura del agua desencadenan el ER. Qin et al. (2014) encontraron que, en respuesta a las perturbaciones submareales y al estrés, $Z$. marina aumentó la producción de semillas y el ER. Esta tendencia se ha descrito para otros pastos marinos (Cabaço y Santos 2012), pero no fue el caso para Z. marina a $-1.90 \mathrm{~m}$ en BSQ.

La longitud y la densidad de los haces vegetativos y reproductivos no fueron significativamente diferentes entre años. La longitud y la densidad no fueron afectadas por la temperatura, pero estuvieron relacionadas significativamente con la profundidad. Se encontraron haces más largos en sitios más profundos, con un efecto negativo en la densidad de haces; 
environmental conditions and increase survival or reproduction. In plants RE accounts for most of the energy a population invests in sexual reproduction (Klinkhamer et al. 1990). Zostera marina showed lower RE values in the subtidal environment. At subtidal depths, available energy goes mainly to vegetative shoot reiteration, while in the intertidal environment, a larger amount of energy is channeled to sexual reproduction, as evidenced by the differences in the number of reproductive shoots in each zone. Zostera marina has been shown to increase its RE over a stress gradient or in a disturbed area (Cabaço and Santos 2012, Qin et al. 2014, Blok et al. 2018).

The perennial populations of $Z$. marina in subtidal and intertidal environments at SQB seem to be well adapted to the local conditions. Their best reproductive strategy is to produce vegetative lateral shoots year-round. Nonetheless, sexual reproduction is important for maintaining the species' genetic variability, representing a potential tool to insure persistence through a seed bank during catastrophic environmental events: ENSO, extraordinary storms, or climate change.

Seagrass habitats are declining (Cabello-Pasini et al. 2003). Thus, it is important to keep seagrass habitats in good health by maintaining or improving their conservation status. We believe there is a stress gradient that increases from the subtidal to the intertidal environment, with reproductive structures being longer in the subtidal environment as a response to the less stressful environmental conditions. Stress is mainly caused by desiccation due to air exposure and high surface water temperatures. The second most important stress factor would be light intensity at the intertidal level.

Resources are allocated to attain more or faster reproductive growth under harsher conditions (Cabaço and Santos 2012). We recorded lower RE rates in the subtidal environment than in the intertidal environment as a result of less energy being invested in sexual reproduction (Figs. 4, 5). Energy in the subtidal environment is used to increase vegetative shoot density. The sexual life cycle of $Z$. marina in SQB was shorter in the subtidal environment.

Eelgrass ecosystem structure and function are shifting because of drivers that range from local to global scales. Zostera marina meadows are declining in distribution and total area (Cabello-Pasini et al. 2003), and these changes are attributed to coastal degradation and climate change. These changes are not completely understood, nor do we know what coastal areas will be affected in the short and long terms. In SQB water turbidity has increased by 2-fold in the last 2 decades (Cabello-Pasini et al. 2003). As a consequence, the number of subtidal $Z$. marina meadows has decreased, and shallower intertidal mudflats have been colonized (Cabello-Pasini et al. 2003). Echavarria-Heras et al. (2006) concluded that the 1986-1987 ENSO event had a negative impact on the SQB eelgrass meadows. Solana-Arellano et al. (2008) mentioned the negative effects
Nielsen et al. (2002) y Henderson y Hacker (2015) encontraron resultados similares. La duración del ciclo reproductivo, el momento de aparición de los haces reproductivos y el desprendimiento de los haces varían con respecto a la luz o la temperatura (Johnson et al. 2003, Lee et al. 2005). Encontramos diferencias entre años en biomasa, pero no en densidad. Patrones semejantes fueron descritos por Olesen y Sand-Jensen (1994) para poblaciones sujetas a severas reducciones de biomasa, donde la biomasa estuvo asignada principalmente a la producción de haces laterales.

El cambio climático está cambiando los intervalos de distribución de los taxones, las comunidades y los ecosistemas, y la dinámica de las poblaciones que habitan los márgenes latitudinales de estos intervalos de distribución será de suma importancia para determinar las respuestas de las especies al cambio climático (Hampe y Petit 2005). La BSQ es una laguna con baja radiación incidente y alta turbidez (Nielsen et al. 2002, Cabello-Pasini et al. 2003), donde la tendencia de la temperatura estacional tiene un efecto importante en la biomasa y el crecimiento (IbarraObando et al. 1997, Poumian-Tapia e Ibarra-Obando 1999, Cabello-Pasini et al. 2003). Observamos la misma tendencia durante nuestro estudio, alta biomasa en verano y baja en invierno (Figs. 1-3). Encontramos que la biomasa vegetativa foliar incrementó en más de 4 veces en 1996-1997 $\left(187.5 \pm 79.7 \mathrm{~g}\right.$ PS $\left.\cdot \mathrm{m}^{-2}\right)$ en comparación con la encontrada durante condiciones normales en 1999-2000 $(40.0 \pm 1.6 \mathrm{~g}$ $\mathrm{PS} \cdot \mathrm{m}^{-2}$ ) (Cabello-Pasini et al. 2003) y durante el ENOS de 1992-1993 (57.02 g PS $\cdot \mathrm{m}^{-2}$ ) (Echavarria-Heras et al. 2006). La mayoría de estas diferencias podrían atribuirse a los efectos del ENOS, como lo demuestran los bajos valores de biomasa durante el ENOS de 1986-1987 (Ibarra-Obando et al. 1997). La baja biomasa reportada por Ibarra-Obando et al. (1997) durante el ENOS de 1986-1987 está subestimada, ya que solo analizaron la biomasa foliar del intermareal. En nuestro estudio, la densidad de los haces en el intermareal fue de $889.6 \pm 152.7$ haces $\cdot \mathrm{m}^{-2}$, la cual es similar al valor de 400-1,390 haces $\cdot \mathrm{m}^{-2}$ encontrado durante el ENOS de 19911992 por Echavarria-Heras et al. (2006) y es ligeramente diferente al valor de $695 \pm 54$ haces $\cdot \mathrm{m}^{-2}$ encontrado en 1999-2000 (años no ENOS) por Cabello-Pasini et al. (2003). Los resultados de estos estudios muestran que la densidad de haces no se vio afectada significativamente por los eventos ENOS.

Las estrategias reproductivas pueden resultar de la asignación óptima de recursos entre el crecimiento vegetativo y la reproducción sexual (Freitas-Coelho et al. 2005), donde se establece un equilibrio entre la biomasa vegetativa y la reproductiva para adaptarse a las condiciones ambientales y aumentar la supervivencia o la reproducción sexual. El ER de las plantas representa la mayor parte de la energía que una población invierte en la reproducción sexual (Klinkhamer et al. 1990). Zostera marina mostró valores de ER más bajos en el submareal. A esta profundidad, la energía disponible se destina principalmente a la reiteración de haces vegetativos, mientras que una mayor cantidad de energía se destina a la 
of the 1982 ENSO event in Punta Banda Estuary, $160 \mathrm{~km}$ north of SQB, and Johnson et al. (2003) reported that off San Diego, California (USA), the 1997-1998 ENSO event had a negative impact on $Z$. marina but a positive one on Ruppia maritima. The extreme 1997 ENSO event probably caused the $64.2 \%$ decrease in $Z$. marina biomass as water temperature increased by $1.5^{\circ} \mathrm{C}$, compared with the $0.25^{\circ} \mathrm{C}$ increase associated with other ENSO events (Trenberth et al. 2002). The warmer water conditions increased stress in the environment and forced a $53.8 \%$ increase in RE. Blok et al. (2018) suggested that warmer waters could increase the capacity for sexual reproduction at northern latitudes where the waters are colder. This condition reduced vegetative biomass and allowed $R$. maritima to invade new spaces and displace or stop the expansion of Z. marina (Johnson et al. 2003). The increase in sexual reproduction in Z. marina could be associated with its ability to adapt to new environments and it could enable ecological specialization (Hadany and Otto 2009). It has been suggested that sexual reproduction is an important adaptation mechanism in stressful environments that forces plants to produce seeds in large quantities (Cadenasso et al. 2003), increasing the seed bank and allowing the invasion of new spaces. The evolution of sexual reproduction could be an adaptive strategy to cope with environmental stress and it could be associated with specializations for living under stress. Specialization in a specific stressful environment does not imply that a population will perform well when confronted with other environmental stressors (Hadany and Otto 2009).

We don't know for how long or which consequences the Z. marina ecosystem will face when affected by the seemingly unpredictable trends in climate change. There are other stressors not considered here: water turbidity, scarce nutrient flux, light attenuation, and human impacts. These stressors can act alone or in conjointly (Cabello-Pasini et al. 2003, Echavarria-Heras et al. 2006). Also, the result of competition with invaders like $R$. maritima is uncertain. In Chesapeake Bay, Shields et al. (2018) showed that the density of $Z$. marina exhibited periods of decline followed by recovery, increased reproductive output, and regrowth after water temperature and other water parameters improved but that the invading $R$. maritima dominated many areas of the bay previously dominated by $Z$. marina, posing a threat to the survival of $Z$. marina.

Our data suggests that eelgrass living at $-1.90 \mathrm{~m}$ produce more vegetative biomass but are less sexually active, that shoots between -0.05 and +0.01 m produce vegetative biomass and are sexually active, and that shoots at +0.10 and $+0.20 \mathrm{~m}$ show high sexual reproduction. Intertidal Z. marina showed higher vegetative shoot density and RE, while subtidal Z. marina showed high vegetative biomass and long shoots. Shoots on boundary edges are continuously under stress and will probably be the most drastically affected by climate change and catastrophic events. reproducción sexual en el intermareal, como fue evidenciado por las diferencias en el número de haces reproductivos en cada zona. Se ha confirmado que Z. marina aumenta su ER en un gradiente de estrés o en un área perturbada (Cabaço y Santos 2012, Qin et al. 2014, Blok et al. 2018).

Las poblaciones perenes de Z. marina en el submareal y el intermareal de BSQ parecen estar bien adaptadas al ambiente local. Su mejor estrategia reproductiva es producir haces laterales vegetativos durante todo el año. No obstante, la reproducción sexual es importante para mantener la variabilidad genética de la especie, lo que representa una estrategia potencial para asegurar la persistencia de la especie a través de un banco de semillas durante eventos ambientales catastróficos: ENOS, tormentas extraordinarias o cambio climático.

Los hábitats de pastos marinos están disminuyendo (Cabello-Pasini et al. 2003). Por lo tanto, es importante mantener estos ambientes en buen estado de salud manteniendo o mejorando su estado de conservación. Consideramos que hay un gradiente de estrés que aumenta del submareal al intermareal y que las estructuras reproductivas fueron más largas en el submareal, dadas las condiciones menos estresantes del ambiente. El estrés es causado principalmente por la desecación debida a la exposición al aire y a las altas temperaturas del agua superficial. El segundo factor de estrés más importante es la intensidad de la luz en el intermareal.

Los recursos energéticos se asignan para producir un crecimiento reproductivo mayor o más rápido en condiciones más adversas (Cabaço y Santos 2012). En este estudio registramos tasas de ER más bajas en el submareal que en el intermareal, producto de una menor inversión de energía en la reproducción sexual (Figs. 4, 5). En el submareal, la energía se utiliza principalmente para incrementar la densidad de haces vegetativos. El ciclo reproductivo de Z. marina en BSQ fue más corto en el submareal.

La estructura y la función de los ecosistemas del pasto marino están cambiando debido a las presiones de escalas desde locales hasta globales. Las praderas de Z. marina están disminuyendo en distribución y área total (Cabello-Pasini et al. 2003), y estos cambios se atribuyen a la degradación costera y al cambio climático. Estos cambios no se comprenden del todo y tampoco se sabe qué zonas costeras se verán afectadas a corto y largo plazo. En BSQ, la turbidez del agua se ha duplicado en las últimas 2 décadas (Cabello-Pasini et al. 2003). Como consecuencia, el número de praderas submareales de Z. marina ha disminuido y las marismas intermareales más someras han sido colonizadas (Cabello-Pasini et al. 2003). Echavarria-Heras et al. (2006) concluyeron que el ENOS de 1986-1987 tuvo un impacto negativo en las praderas del pasto marino en BSQ. Solana-Arellano et al. (2008) mencionaron los efectos negativos del ENOS de 1982 en el estuario de Punta Banda, $160 \mathrm{~km}$ al norte de BSQ, y Johnson et al. (2003) mencionaron que, frente a San Diego, California, el ENOS de 1997-1998 tuvo un impacto negativo en Z. marina, pero positivo en Ruppia maritima. Las condiciones extremas del 


\section{ACKNOWLEDGMENTS}

The National Council for Science and Technology (Mexico) funded project C01-1434.

\section{REFERENCES}

Bertelli CM, Unsworth RKF. 2018. Light stress responses by the eelgrass, Zostera marina (L). Front Environ Sci. 6:39. https://doi.org/10.3389/fenvs.2018.00039

Blok SE, Olesen B, Krause-Jensen D. 2018. Life history events of eelgrass Zostera marina L. populations across gradients of latitude and temperature. Mar Ecol Prog Ser. 590:79-93. https://doi.org/10.3354/meps12479

Cabaço S, Santos R. 2012. Seagrass reproductive effort as an ecological indicator of disturbance. Ecol Indic. 23:116-122. https://doi.org/10.1016/j.ecolind.2012.03.022

Cabello-Pasini A, Lara-Turrent C, Zimmerman RC. 2002. Effect of storms on photosynthesis, carbohydrate content and survival of eelgrass populations from a coastal lagoon and the adjacent open ocean. Aquat Bot. 74(2):149-164. https://doi.org/10.1016/S0304-3770(02)00076-1

Cabello-Pasini A, Muñiz-Salazar R, Ward DH. 2003. Annual variations of biomass and photosynthesis in Zostera marina at its southern end of distribution in the North Pacific. Aquat Bot. 76(1):31-47.

https://doi.org/10.1016/S0304-3770(03)00012-3

Cadenasso ML, Pickett STA, Weathers KC, Jones CG. 2003. A framework for a theory of ecological boundaries. BioScience. 53(8):750-758

https://doi.org/10.1641/0006-3568(2003)053[0750:AFFATO]2.0 .CO;2

[CICESE] Centro de Investigación Científica y de Educación Superior de Ensenada. 2019. Estaciones Mareográficas y Meteorológicas Costeras. Ensenada (Baja California, Mexico): Laboratorio del Nivel del Mar de CICESE; [accessed 2019 March]

h t t p://redmar.cicese.mx/e m m c/web m/e m m c. php?nvar $=$ met\&nsta $=$ SNQN\&nday $=2 \& R=1$

Echavarria-Heras HA, Solana-Arellano E, Franco-Vizcaíno E. 2006. The role of increased sea surface temperature on eelgrass leaf dynamics: onset of El Niño as a proxy for global climate change in San Quintín Bay, Baja California. Bull Southern California Acad Sci. 105(3):113-127. https://doi.org/10.3160/0038-3872(2006)105[113:TROISS]2.0.CO;2

Freitas-Coelho F, Deboni L, Santos-Lopes F. 2005. Density-dependent reproductive and vegetative allocation in the aquatic plant Pistia stratiotes (Araceae). Rev Biol Trop. 53(3-4):369-376. https://doi.org/10.15517/rbt.v53i3-4.14599

Hadany L, Otto SP. 2009. Condition-dependent sex and the rate of adaptation. Am Nat. 174(S1):S71-S78. https://doi.org/10.1086/599086

Hammer KJ, Borum J, Hasler-Sheetal H, Shields EC, Sand-Jensen K, Moore KA. 2018. High temperatures cause reduced growth, plant death and metabolic changes in eelgrass Zostera marina. Mar Ecol Prog Ser. 604:121-132. https://doi.org/10.3354/meps 12740

Hampe A, Petit RJ. 2005. Conserving biodiversity under climate change: the rear edge matters. Ecol Lett. 8(5):461-467. https://doi.org/10.1111/j.1461-0248.2005.00739.x

Henderson J, Hacker SD. 2015. Buried alive: an invasive seagrass (Zostera japonica) changes its reproductive allocation in response to sediment disturbance. Mar Ecol Prog Ser. 532:123-136.

https://doi.org/10.3354/meps11335 evento ENOS de 1997 probablemente causaron la disminución del $64.2 \%$ de la biomasa de Z. marina al aumentar en 1.5 ${ }^{\circ} \mathrm{C}$ la temperatura del agua, en comparación con el aumento de $0.25^{\circ} \mathrm{C}$ asociado a otros eventos de ENOS (Trenberth et al. 2002). Las aguas más cálidas aumentaron el estrés en el medio ambiente y forzaron un aumento del $53.8 \%$ en el ER. Blok et al. (2018) sugirieron que aguas más cálidas podrían aumentar la capacidad de reproducción sexual en latitudes más al norte donde las aguas son más frías. Esta condición redujo la biomasa vegetativa y permitió que $R$. maritima invadiera nuevos espacios y desplazara o detuviera la expansión de Z. marina (Johnson et al. 2003). El aumento en la reproducción sexual de Z. marina podría estar asociado a su capacidad de adaptarse a nuevos entornos y podría facilitar la especialización ecológica (Hadany y Otto 2009). Se ha sugerido que la reproducción sexual es una adaptación importante en ambientes estresantes que obliga a las plantas a producir semillas en grandes cantidades (Cadenasso et al. 2003), lo cual aumenta el banco de semillas y permite la invasión de nuevos espacios. Se puede argumentar que la evolución de la reproducción sexual es una estrategia adaptativa al estrés ambiental y podría estar asociada con especializaciones para vivir bajo estrés. La especialización a un entorno estresante específico no implica que una población se desempeñe adecuadamente cuando se enfrente a otros factores ambientales estresantes (Hadany y Otto 2009).

No sabemos por cuánto tiempo o qué consecuencias enfrentará el ecosistema de Z. marina cuando se vea afectado por las tendencias aparentemente impredecibles del cambio climático. Hay otros factores estresantes que no se consideraron aquí: la turbidez del agua, el escaso flujo de nutrientes, la atenuación de la luz y los impactos humanos. Estos factores de estrés pueden actuar solos o en conjunto (Cabello-Pasini et al. 2003, Echavarria-Heras et al. 2006). Además, el resultado de la competencia con invasores como $R$. maritima es incierto. En la bahía de Chesapeake, Shields et al. (2018) encontraron que la densidad de Z. marina mostró periodos de disminución seguidos de periodos de recuperación, aumento en la producción reproductiva y resurgimiento después de que la temperatura del agua y otros parámetros del agua mejoraron, pero la especie invasora, $R$. maritima, dominó muchas áreas de la bahía previamente dominadas por $Z$. marina, lo cual representa un riesgo para la supervivencia de Z. marina.

Nuestros datos sugieren que el pasto marino a $-1.90 \mathrm{~m}$ produce más biomasa vegetativa, pero es menos activo sexualmente; los haces localizados entre -0.05 y $+0.01 \mathrm{~m}$ producen biomasa vegetativa y son sexualmente activos; y los haces entre $+0.10 \mathrm{y}+0.20 \mathrm{~m}$ tienen una alta reproducción sexual. En el intermareal, Z. marina tuvo mayor densidad de haces vegetativos y mayor ER, mientras que en el submareal tuvo mayor biomasa vegetativa y haces más largos. Los haces en los límites de los bordes están continuamente sometidos a estrés y probablemente serán los más afectados por el cambio climático y cualquier otro evento catastrófico. 
Hernández-Carmona G, Riosmena-Rodríguez R, ServiereZaragoza E, Ponce-Díaz G. 2011. Effect of nutrient availability on understory algae during El Niño Southern Oscillation (ENSO) conditions in Central Pacific Baja California. J Appl Phycol. 23(3):635-642. https://doi.org/10.1007/s10811-011-9656-5

Ibarra-Obando SE, Boudouresque CF, Roux M. 1997. Leaf dynamics and production of a Zostera marina bed near its southern distributional limit. Aquat Bot. 58(2):99-112. https://doi.org/10.1016/S0304-3770(97)00020-X

Johnson MR, Williams SL, Lieberman CH, Solbak A. 2003. Changes in the abundance of the seagrasses Zostera marina L. (eelgrass) and Ruppia maritima L. (widgeongrass) in San Diego, California, following an El Niño Event. Estuaries. 26(1):106115. https://doi.org/10.1007/BF02691698

Kaul V. 2016. Resource allocation in angiosperms. The International Journal of Plant Reproductive Biology. 8(2):166-172.

Klinkhamer PGL, de Jong TJ, Meelis E. 1990. How to test for proportionality in the reproductive effort of plants. Am Nat. 135(2):291-300. https://doi.org/10.1086/285045

Kumar A, Wang W, Hoerling MP, Leetmaa A, Ji M. 2001. The sustained North American warming of 1997 and 1998. J Clim. 14(3):345-353. https://doi.org/10.1175/1520-0442(2001)014<0345:TSNAWO $>2$. $0 . \mathrm{CO} ; 2$

Lee SY, Kim JB, Lee SM. 2006. Temporal dynamics of subtidal Zostera marina and intertidal Zostera japonica on the southern coast of Korea. Mar Ecol. 27(2):133-144. https://doi.org/10.1111/j.1439-0485.2006.00089.x

Lee SY, Lee SM, Choi CI. 2005. Phenology and reproductive effort of two Zostera marina L. populations on the southern coast of Korea. Ocean Polar Res. 27(1):67-74. https://doi.org/10.4217/opr.2005.27.1.067

Nielsen SL, Sand-Jensen K, Borum J, Geertz-Hansen O. 2002. Depth colonization of eelgrass (Zostera marina) and macroalgae as determined by water transparency in Danish coastal water. Estuaries. 25(5):1025-1032. https://doi.org/10.1007/BF02691349

Olesen B, Sand-Jensen K. 1994. Biomass-density patterns in the temperate seagrass Zostera marina. Mar Ecol Prog Ser. 109:283-291. https://doi.org/10.3354/meps109283

Phillips RC, McMillan C, Bridges KW. 1983. Phenology of eelgrass, Zostera marina L., along latitudinal gradients in North

\section{Agradecimitentos}

El Consejo Nacional de Ciencia y Tecnología financió el proyecto $\mathrm{C} 01-1434$.

America. Aquat Bot. 15:145-156. https://doi.org/10.1016/0304-3770(83)90025-6

Poumian-Tapia M, Ibarra-Obando SE. 1999. Demography and biomass of the seagrass Zostera marina in a Mexican coastal lagoon. Estuaries. 22(4):837-847. https://doi.org/10.2307/1353065

Qin LZ, Li WT, Zhang XM, Nie M, Li Y. 2014. Sexual reproduction and seed dispersal pattern of annual and perennial Zostera marina in a heterogeneous habitat. Wetl Ecol Manag. 22(6):671682.

https://doi.org/10.1007/s11273-014-9363-5

Riosmena-Rodríguez R, Muñiz-Salazar R, López-Calderón J, Torre-Cosio J, Meling A, Talbo SL, Sage GK, Ward DH, Cabello-Pasini A. 2013. Conservation status of Zostera marina populations at Mexican Pacific. Chapter 2. In: Daniels JA (ed.), Advances in Environmental Research. Vol. 27. Florida (USA): Nova Science Publishers. p. 35-63.

Sexton JP, McIntyre PJ, Angert AL, Rice KJ. 2009. Evolution and ecology of species range limits. Annu Rev Ecol Evol Syst. 40(1):415-436.

https://doi.org/10.1146/annurev.ecolsys.110308.120317

Shields EC, Moore KA, Parrish DB. 2018. Adaptations by Zostera marina dominated seagrass meadows in response to water quality and climate forcing. Diversity. 10(4):125. https://doi.org/10.3390/d10040125

Solana-Arellano E, Echavarria-Heras H, Franco-Vizcaíno E. 2008. An economical non-destructive method for estimating eelgrass, Zostera marina (Potamogetonaceae) leaf growth rates: formal development and use in northwestern Baja California. Rev Biol Trop. 56(3):1003-1013. https://doi.org/10.15517/rbt.v56i3.5688

Trenberth KE, Caron JM, Stepaniak DP, Worley S. 2002. Evolution of El Niño-Southern Oscillation and global atmospheric surface temperatures. J Geophys Res. 107(D8):AAC 5-1-AAC 5-17.

https://doi.org/10.1029/2000JD000298

Zar JH. 2010. Biostatistical Analysis. 5th ed. New Jersey (USA): Pearson Prentice Hall. 964 p.

Received 4 February 2020, accepted 20 June 2020. 